\title{
Stepwise Optimization Model of Mooring System Based on Mechanics Analysis and Dichotomy
}

\author{
Jian Liu \\ School of North China Electric Power University, Baoding 071000, China \\ 1766937377@qq.com
}

Keywords: Mooring system, Mechanics analysis, Dichotomy, Stepwise optimization.

\begin{abstract}
The extreme motion area, draft depth, steel pipe The extreme motion area, draft depth, steel pipe tilt angle and other parameters of the buoy are solved step-by-step with taking more parameters into account: single parameter of wind speed, double parameters of wind speed and heavy ball quality, multi parameters of wind speed, heavy ball quality, wave impact force and sea water depth. Firstly, the stress of the joint is decomposed. Then combining the solving condition that the vertical height between the anchor and the sea surface is equal to the water depth, according to the dichotomy, we can approximately get the inclination angle of steel pipe and steel drum and the swimming area and draft depth of the buoy under the situations that the angle between the bottom of the chain and the horizontal line is less than 16 degrees, the angle between the steel drum and the vertical line is less than 5 degrees, the wind speed is $12 \mathrm{~m} / \mathrm{s}, 14 \mathrm{~m} / \mathrm{s}, 36 \mathrm{~m} / \mathrm{s}$. With the increase in wind speed, we found that the angle between the steel drum and the vertical line does not meet the conditions, and we need to increase the quality of steel balls and change the anchor chain model to adjust the tilt angle of steel drum. Finally, in the case of high wind speed, the ball quality should be at least $1928.07 \mathrm{~kg}$ or at most $4531.64 \mathrm{~kg}$.
\end{abstract}

\section{Introduction}

In this paper, according to Newton mechanics, the steel pipe, steel drum, heavy ball, welding anchor chain and special anti-drag anchor the buoy system and mooring system of were respectively analyzed by force.By the force balance, we can get the relationships between the pull、 tilt angle of each chain, steel drum, steel pipes, buoys and the buoyant draft depth.Moreover, the other parameters waiting to be solved are identically expressed with buoy draft depth $h$, and further the relationship between the buoyant draft depth $\mathrm{h}$ and seawater depth $\mathrm{h} 0$ is got. The buoyant draft depth is solved by the depth of the sea water.Because of the difficulty in solving the buoyant depth $h$ of the buoy, this paper adopts the least dichotomy to approximate the optimal solution. Finally, the one-dimensional equation for the buoyant draft depth based on the known seawater depth is listed, which is solved using matlab.

\section{Analysis of Model}

In order to solve the tilt angleof the steel pipe, anchor chain shape, draft depth and swimming area of the buoy, the force analysis for each part is carried out using Newtonian mechanics and torque. Combined with the information given by the subject, the function or equation of the relationships between the variables is fiannly confirmed.

(1) Firstly, the force analyses of the anchor and buoy are carried out as the follows:

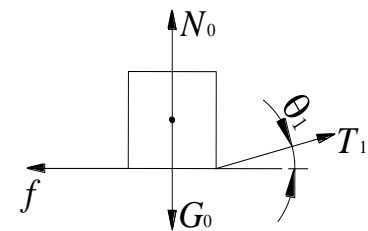

Figure 1 Force Analysis of Anchor

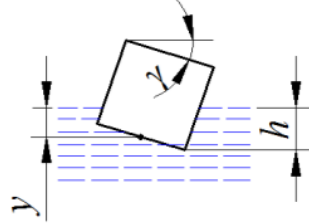

Figure 2 Force Analysis of Buoy 
From the force analysis of the whole excluding the part of anchor, it can be seen that the relationship between the pull and the force of the wind in the horizontal direction submits to the followed function.

$$
F_{x}=T_{1} \cos \phi_{1}
$$

As shown in Figure 1, the buoy has a normal area in the vertical direction of the wind,which can be expressed as the function belowed:

$$
S=\frac{\frac{1}{2} \pi r^{2}}{\cos \gamma} \cos \phi+2 r \times\left(d-\frac{h}{\cos \gamma}+r \tan \gamma\right) \cos (\gamma-\phi)+\frac{1}{2} \pi r^{2} \sin (\gamma-\phi)
$$

After the decompositon of the wind along the vertical and heritical directions, the buoyancy of the buoy is work out as follows:

$$
\begin{aligned}
& F_{\mathrm{x}}=0.625 v^{2} S \cos \phi \\
& F_{\mathrm{y}}=0.625 v^{2} S \sin \phi \\
& F_{b}=\rho \pi r^{2} g\left(\frac{n}{\cos \gamma}-r \tan \gamma\right)
\end{aligned}
$$

(2) Do the moment analysis of one chain in the anchor as shown in figure 3:

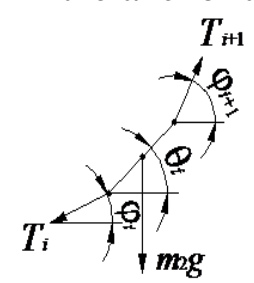

Figure 3 Force Analysis of Anchor Chain

The buoyancy of the anchor chain is negligible. For one of its chain, the moment balance equation around its end point is :

$$
\frac{1}{2} m g l_{i} \cos \theta_{i}=T_{i+1} l_{i} \sin \left(\phi_{i+1}-\theta_{i}\right) \quad 1 \leq i \leq 210
$$

The answer to this equation is:

$$
\theta_{i}=\frac{1}{\sqrt{1+\left(\frac{T_{i+1} \sin \phi_{i+1}}{\frac{1}{2} m g+T_{i} \cos \phi_{i+1}}\right)^{2}}}
$$

For steel drums and steel pipes, the sum of the moments around the upper end point is also zero, noting that there is buoyancy in the vertical direction, then their torque balance equations are as follows:

For the steel drum, when $i=212$, the equation is:

$$
\frac{1}{2}\left(m g-\rho \pi r_{3}^{2} d g\right) l_{i} \cos \theta_{i}=T_{i+1} l_{i} \sin \left(\phi_{i+1}-\theta_{i}\right), \quad \mathrm{i}=212
$$

For the steel pipe, the equation is:

$$
\frac{1}{2}\left(m g-\rho \pi r_{2}^{2} d g\right) l_{i} \cos \theta_{i}=T_{i+1} l_{i} \sin \left(\phi_{i+1}-\theta_{i}\right), \quad 211<=\mathrm{i}<=215
$$


For the anchor chain part, because of its balance in both the vertical direction and the horizontal direction, the following equations can be got:

$$
\begin{aligned}
& F_{x}=T_{i} \cos \phi_{i} \\
& F_{b}-m_{b} g+\rho \pi r_{3}^{2} d g-m_{t} g+4\left(\rho \pi r_{2}^{2} d g-m_{t} g\right)-F_{y}-(211-i) m g=T_{i} \sin \phi_{i}
\end{aligned}
$$

According to the equations $(11) /(12)$, the solutions are:

$$
\begin{aligned}
& T_{i}=\sqrt{F_{x}^{2}+\left[-(211-\mathrm{i}) \mathrm{mg}+\rho \pi r_{3}^{2} d g+4 \rho \pi r_{2}^{2} d g-F_{\mathrm{y}}-m_{t} g-4 m_{g} g-m_{q} g-m_{b} g\right]^{2}} \\
& \phi_{i}=\cos ^{-1} \frac{F_{x}}{T_{i}}
\end{aligned}
$$

where $i$ is between 1 and 211 .

Orthogonal decomposition of steel pipes and steel drums is analyzed only by a buoyancy in the vertical direction, which can be similarly obtained:

where $i$ is between 212 and 216 .

$$
\begin{aligned}
T_{i} & =\sqrt{F_{x}^{2}+\left[-210 \mathrm{mg}+\rho \pi r_{3}^{2} d g+(216-i)\left(\rho \pi r_{2}^{2} d g-F_{\mathrm{y}}-m_{g} g\right)-m_{b} g\right]^{2}} \\
\phi_{i} & =\cos ^{-1} \frac{F_{x}}{T_{i}}
\end{aligned}
$$

\section{The Establishment and Solution of Model}

For the moment analysis of the first chain, taking the upper node as the fulcrum, conbining the equations (2)(3)(4)(5), the expression of the draft depth $h$ can be obtained:

where $i$ is between 1 and 211 .

$T_{i}=\sqrt{F_{x}^{2}+\left[-(211-\mathrm{i}) \mathrm{mg}+\rho \pi r_{3}^{2} d g+4 \rho \pi r_{2}^{2} d g-F_{\mathrm{y}}-m_{t} g-4 m_{g} g-m_{\mathrm{q}} g-m_{\mathrm{b}} g\right]^{2}}$

where $\mathrm{i}$ is between 212 and 216 .

$$
T_{i}=\sqrt{F_{x}^{2}+\left[-210 \mathrm{mg}+\rho \pi r_{3}^{2} d g+(216-i)\left(\rho \pi r_{2}^{2} d g-F_{\mathrm{y}}-m_{g} g\right)-m_{b} g\right]^{2}}
$$

From the above equations, it is easy to get the angle between each chain of anchor, steel drum, steel pipe and horizontal line.

$$
\left\{\begin{array}{l}
\theta_{i}=\frac{1}{\sqrt{1+\left(\frac{T_{i+1} \sin \phi_{i+1}}{\frac{1}{2} m g+T_{i} \cos \phi_{i+1}}\right)^{2}}} \\
\theta_{i}=\frac{1}{\sqrt{1+\left(\frac{T_{i+1} \sin \phi_{i+1}}{\frac{1}{2}\left(m_{g} g-\rho \pi r_{3}^{2} d g\right)+T_{i} \cos \phi_{i+1}}\right)^{2}}} \\
\theta_{i}=\frac{1}{\sqrt{1+\left(\frac{1}{\frac{1}{2}\left(m_{g} g-\rho \pi r_{2}^{2} d g\right)+T_{i} \cos \phi_{i+1}}\right)^{2}}}
\end{array}\right.
$$

As the positive solution is more difficult, here the draft depth and buoy tilt angle are derived from the depth of sea water in opposite direction. The angle between the various parts of the mooring system and the horizontal direction has been obtained from the above equation, therefore, the above equation can be also used in the vertical direction:

$$
\sum_{i=1}^{215} l_{i} \sin \phi_{i}+h-r \sin \gamma=18 \mathrm{~m}
$$


When the wind speed is $12 \mathrm{~m} / \mathrm{s}, 24 \mathrm{~m} / \mathrm{s}$ and $36 \mathrm{~m} / \mathrm{s}$ respectively, the inclination angle of steel drum and steel pipe, the inclination angle of each chain of anchor, the limit swimming area of buoy can be soled using dichotomy.

Table 1. Solutions of the steel drum, tilt angles of four steel pipe and limit radius

\begin{tabular}{c|c|c|c|c|c|c}
\hline $\begin{array}{c}\text { Wind } \\
\text { Speed }\end{array}$ & $\boldsymbol{\theta}_{212}$ & $\boldsymbol{\theta}_{213}$ & $\boldsymbol{\theta}_{214}$ & $\boldsymbol{\theta}_{215}$ & $\theta_{t}$ & $\begin{array}{c}\text { Limit } \\
\text { Radius r }\end{array}$ \\
\hline $12 \mathrm{~m} / \mathrm{s}$ & $0.7854^{\circ}$ & $0.7801^{\circ}$ & $0.7612^{\circ}$ & $0.7532^{\circ}$ & $0.7954^{\circ}$ & $9.623 \mathrm{~m}$ \\
\hline $14 \mathrm{~m} / \mathrm{s}$ & $1.5954^{\circ}$ & $1.5936^{\circ}$ & $1.5898^{\circ}$ & $1.5879^{\circ}$ & $2.313^{\circ}$ & $17.567 \mathrm{~m}$ \\
\hline $36 \mathrm{~m} / \mathrm{s}$ & $2.3561^{\circ}$ & $2.3553^{\circ}$ & $2.3503^{\circ}$ & $2.35^{\circ}$ & $7.884^{\circ}$ & $34.2114 \mathrm{~m}$ \\
\hline
\end{tabular}

\section{Conclusion}

When the wind speed is $12 \mathrm{~m} / \mathrm{s}$ and $14 \mathrm{~m} / \mathrm{s}$ the angle between the steel drum and the vertical direction is $0.7954^{\circ}$ and $2.313^{\circ}$ respectively. The angles between four steel pipes and the vertical direction is incremented by $0.7854^{\circ}$ and $1.5879^{\circ}$ from top to down. The buoy limit travel area is a radius of $9.623 \mathrm{~m}$, a circle of $17.567 \mathrm{~m}$, and the draft depth are $0.7592 \mathrm{~m}$ and $0.7945 \mathrm{~m}$ respectively. The angle between the 210 chain nodes and the horizontal direction is incremented by $10.078^{\circ}$ and $9.114^{\circ}$ respectively. When the wind speed is $36 \mathrm{~m} / \mathrm{s}$, only the weight of the heavy ball are in the range of $1928.07 \mathrm{~kg}$ $4531.14 \mathrm{~kg}$, can the buoy not be immersed in the water as a whole, ensuring that the tilt angles at the bottom of the steel drum and anchor meet the requirements.

\section{References}

[1]. Huoming Zhang,Ju Fan,Jianmin Yang. Study on Fast Calculation Method of Static Characteristics of Deep Water Mooring System [J]. Sailing Engineering,2007,02:64-68.

[2]. Long Yu,Jiahua Tan. Study on Optimum Design and Application of Multi - component Suspension Cable Mooring System in Deep Water [J]. Journal of East China Shipbuilding Institute (Natural Science Edition),2004,05:8-13.

[3]. Bo Tong,Jianmin Yang,Xin Li. Coupled Dynamic Analysis of Submarine Mooring System with Deep Submersible Submarine [J]. China Ocean Platform,2008,06:1-7. 